\section{Fast Parametric Modeling of Radio Astronomy Reflector Antenna Noise Temperature}

\author{
Dirk I. L. de Villiers, Senior Member, IEEE
}

\begin{abstract}
This communication presents an approximate method to rapidly estimate the antenna noise temperature of a reflector antenna system as a function of the geometric parameters describing the reflector dishes. The initial estimate is established by neglecting the main reflector from the calculation domain, thus speeding up the calculations significantly. The accuracy may be improved by aligning the estimated results with those from full model simulations, performed at a limited set of points scattered through the parameter space, by a linear regression correction on the residuals. Results of applications on a variety of reflector configurations for some commonly used parameter sets confirm the accuracy of the method to be better than $1 \%$, with speed-ups of more than an order of magnitude typical.
\end{abstract}

Index Terms-Noise, radio astronomy, reflector antennas.

\section{INTRODUCTION}

Antenna noise temperature is an important performance metric in the design of several types of ground based reflector antenna systems - most notably ground station antennas for deep space communication [1] and radio telescope antennas [2], [3]. Specifically the receiving sensitivity, which is the ratio of effective area and noise temperature of the antenna system, is of interest, with maximization of this metric often being the primary objective of ground based reflector antenna designs. The most time consuming part of the calculation of the receiving sensitivity typically lies in finding the antenna noise temperature, since this requires the calculation (or measurement) of the radiation pattern of the reflector system over the full $4 \pi$ steradian sphere, and a subsequent integration of the weighted pattern. An early example of a reflector system optimized for receiving sensitivity is reported in [4]. To make the repeated calculation of the radiation pattern (including feed dish interactions) required for the optimization tractable, this example used a custom multiple reflection approach.

The development of modern radio telescope systems, such as MeerKAT [2] and SKA [3], has sparked renewed interest in finding fast and reliable ways of calculating antenna noise temperature for use in optimization routines to design these systems. Recent publications [5]-[8] have focused on rapid estimation of antenna noise temperature in offset Gregorian reflector configurations, since this is the choice for the MeerKAT and SKA reflector optics. In all cases the basic premise used to increase the calculation speed is that the main reflector can be removed from the calculation domain - if a suitable correction is applied to the field radiated towards the missing main reflector. The significant speed-up obtained in this way is attributed to the reduced size of the electromagnetic (EM)

Manuscript received xxx xx, 2015; revised xxx xx, 201x; accepted xxx xx, 201x. Date of publication xxx xx, xxxx; date of current version xxx xx, xxxx.

D. I. L. de Villiers is with the Department of Electrical and Electronic Engineering, Stellenbosch University, Stellenbosch, 7600, South Africa (email:ddv@sun.ac.za)

Digital object identifier $\mathrm{xxx}$ problem, as well as to the slower angular variation in the farfield pattern that is to be integrated (due to the reduced total antenna size). The idea was first presented in [5] (although it has been informally used in the past - see for instance [9]), with a further correction factor added to improve the accuracy over wide bandwidths in [7], [8].

The work in [5]-[8] focused on estimation of the antenna noise temperature as a function of frequency for a specific feed and antenna structure in the offset Gregorian configuration. This communication builds on the idea of omitting the main reflector from the calculation domain to model the antenna noise temperature of a reflector system as a function of the geometric parameters describing the reflectors. Since these parameters are often the inputs to a parameter study or optimization routine, such a computationally cheap parametric model of antenna noise temperature may significantly speed up the process. Accuracy of the model is maintained by casting it into a so-called surrogate based modeling framework [10]. In this context a surrogate model is constructed by aligning a fast to evaluate, but less accurate, coarse model to an accurate, but typically slow to evaluate, fine model. The surrogate model is thus a corrected version of the coarse model, providing high accuracy at low computational cost. In this work the coarse model is the antenna system with the main reflector removed, and the fine model the full reflector system. The alignment (or correction) of the coarse model is done by evaluating the fine model at only a few points in the parameter space, and fitting a regression function through the resulting residuals between the fine and coarse models. This regression function is added to the coarse model as a correction term. Since the underlying coarse model is physically based on the same system as the full fine model, it generalizes well over the parameter space.

The details of the model construction and alignment are described in Section II, and several examples of the method applied to different reflector antenna types (prime focus paraboloid, symmetrical Cassegrain, and shaped offset Gregorian) are provided in Section III. It will be shown that the antenna noise temperature may be accurately modeled - to within less than $1 \%$ error - over wide multi-dimensional parameter spaces, at a fraction of the computational cost required when the full reflector system models are directly evaluated. Lossless and matched antenna structures are assumed throughout, so that the only contribution to the antenna temperature is that from the background radiometric noise.

\section{Surrogate Model Construction}

\section{A. Antenna Noise Temperature Definition}

Antenna noise temperature is calculated from the so called noise temperature integral as [1], [7]

$$
T_{\mathrm{A}}\left(f \mid \boldsymbol{r}_{0}\right)=\frac{\int_{4 \pi} T_{\mathrm{b}}(f, \Omega) P\left(f, \Omega \mid \boldsymbol{r}_{0}\right) d \Omega}{\int_{4 \pi} P(f, \Omega) d \Omega},
$$

where $T_{\mathrm{A}}$ denotes the antenna noise temperature and $P\left(f, \Omega \mid \boldsymbol{r}_{0}\right)$ the total antenna radiation pattern power per unit solid angle, $\Omega$, when pointing in the direction of the unit vector $r_{0}$ at frequency $f$. The brightness temperature distribution of the scene surrounding the antenna is denoted by $T_{\mathrm{b}}(f, \Omega)$, 
which for a rotationally symmetric distribution may be specified in terms of only the polar angle $\theta$ as $T_{\mathrm{b}}(f, \theta)$. A complete description of the brightness temperature model $T_{\mathrm{b}}$ is provided in [11], with some further discussions on different levels of simplification of the model available in [6], [7]. In general, the method descibed in this communication is not limited to the brightness temperature models in [7], [11], and simpler or more complete models may be used if required. The definition in (1) is valid for a single medium and polarization, and it should, in general, be separated to account for the polarization dependent ground emission and scattering as described in [1], [7]. For ease of notation, and without loss of generality, (1) may be written as

$$
T_{\mathrm{A}}\left(f, \theta_{\mathrm{p}}\right)=\int_{4 \pi} T_{\mathrm{b}}\left(f, \Omega_{\mathrm{p}}\right) G(f, \Omega) d \Omega,
$$

where $G$ denotes the antenna gain pattern (since the denominator of (1) represents the total radiated power). The effect of varying elevation (or tipping) angle $\theta_{\mathrm{p}}$ of the system is accounted for by rotating $T_{\mathrm{b}}$ around the $y$-axis by $-\theta_{\mathrm{p}}$, and thus using a rotation matrix to find the mapping $\Omega \rightarrow \Omega_{\mathrm{p}}$. A detailed expansion of the rotation mapping is given in, for instance, [1, Ch. 1], and $\Omega_{\mathrm{p}}$ corresponds to the primed coordinates in [7]. Zenith pointing is aligned with the $z$-axis. Symmetry plane cuts of the three types of reflector systems discussed in this communication are shown in Fig. 1, where some of the important parameters as well as coordinate system definitions are indicated.

\section{B. Calculating Coarse Model Responses}

Following the idea in [5], coarse models of $T_{\mathrm{A}}$, which are physically based on the full models but significantly faster to evaluate, may be constructed by removing the main reflector from the calculation domain. Single reflector systems require calculation of the feed radiation pattern, and in the same way dual reflector systems the calculation of the feed and subreflector radiation pattern. The coarse model gain patterns are indicated as $G^{\mathrm{c}}$, and the resulting antenna temperature as $T_{\mathrm{A}}^{\mathrm{c}}$. In all cases the portion of the pattern $G^{\mathrm{c}}$ radiating towards the main reflector will be assumed to be reflected towards the sky, and the brightness temperature in that region will thus be modified to the sky temperature in the $\theta_{\mathrm{p}}$ direction to give

$$
T_{\mathrm{A}}^{\mathrm{c}}\left(f, \theta_{\mathrm{p}}\right)=\int_{4 \pi} T_{\mathrm{b}}^{\mathrm{c}}\left(f, \Omega_{\mathrm{p}}\right) G^{\mathrm{c}}(f, \Omega) d \Omega,
$$

with

$$
T_{\mathrm{b}}^{\mathrm{c}}\left(f, \Omega_{\mathrm{p}}\right)= \begin{cases}T_{\mathrm{b}}\left(f, \Omega_{\mathrm{p}}\right), & \Omega_{\mathrm{p}}-\Omega^{\mathrm{M}}, \\ T_{\mathrm{b}}\left(f, \theta_{\mathrm{p}}\right), & \Omega_{\mathrm{p}} \cap \Omega^{\mathrm{M}} .\end{cases}
$$

Here standard set notation is used for the solid angle regions $\Omega_{\mathrm{p}}$ and $\Omega^{\mathrm{M}}$, with the definition of the latter illustrated in Fig. 1 as the region, in the radiation pattern coordinate system, subtended by the main reflector. Note that, for the offset Gregorian case, the region indicated by $\Omega^{\mathrm{M}}$ corresponds to the main reflector region indicated in [7, Figs. 5-6] and the mask in [5, Fig. 3(c)].

The fast calculation time of the coarse model is due to the reduced number of unknowns in the calculation space
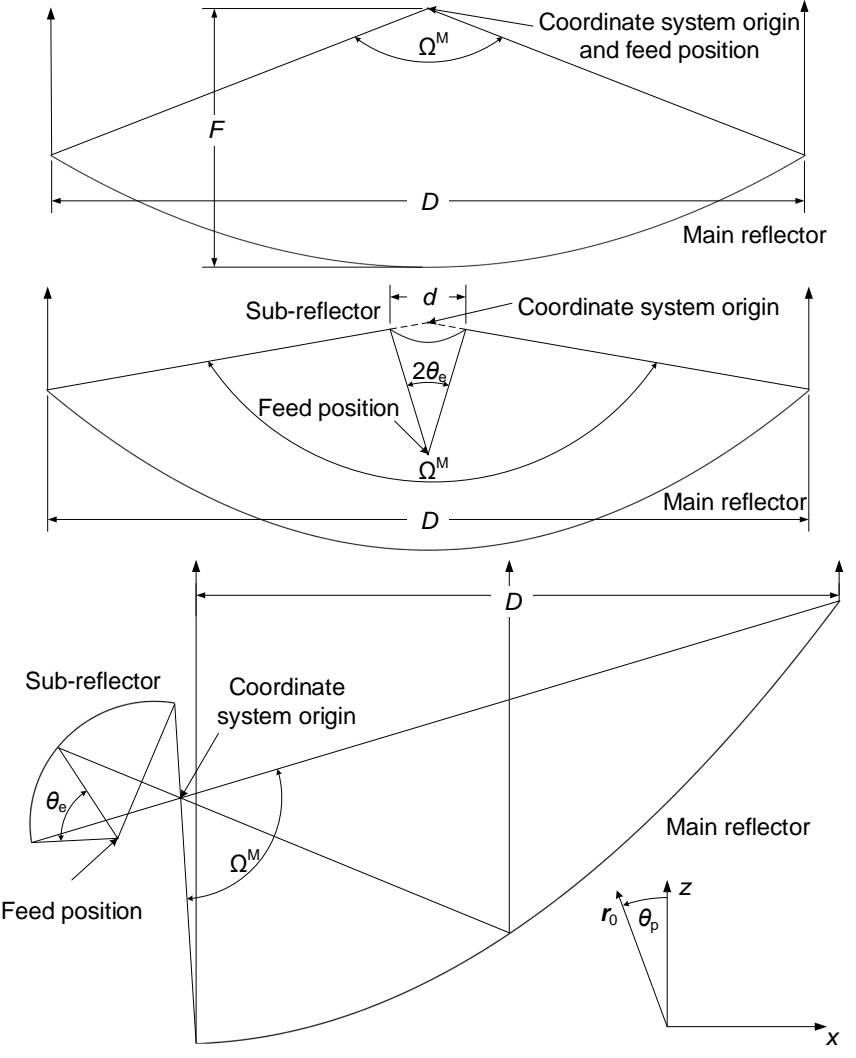

Fig. 1. Symmetry plane cut of a prime focus paraboloid (top), symmetrical Cassegrain (middle), and offset Gregorian (bottom) reflector system. Some of the physical parameters and coordinate system definitions are shown, as well as some of the optical ray paths in the transmit sense. Note that $\Omega^{\mathrm{M}}$ represents a solid angle.

(especially important in full-wave simulations), as well as the slower angular variation of the radiation pattern due to the smaller electrical size of the antenna. The latter effect is particularly obvious when Physical Optics (PO) is used, since the field calculation often becomes a bottleneck in the algorithm when large number of field points are required [12]. Errors in the coarse model arise mainly from from the neglect of any diffraction effects caused by the main reflector. The model thus becomes more accurate with increasing electrical size of the main reflector. A correction factor is suggested in [7], [8] for the offset Gregorian configuration, which improves the predictions for different simulation frequencies. This factor modifies the brightness temperature profile as a function of frequency only, and cannot be easily generalized and extended to a function of the geometric parameters of the system. Here we are interested in changing the physical reflector parameters, so a more general correction strategy, applicable to all reflector geometries, is suggested in Section II-C.

\section{Model Alignment}

To compensate for the inaccuracy inherent in the coarse model, a simple strategy is suggested to construct an improved accuracy surrogate model with little extra computational overhead. The strategy is especially useful when the surrogate model is required over a wide parametric space, as would 
be the case in parametric studies and global optimizations. For each $f$ and $\theta_{\mathrm{p}}$ (suppressed in the following notation) a correction term is added to the coarse model to form the surrogate model $T_{\mathrm{A}}^{\mathrm{s}}$ as

$$
T_{\mathrm{A}}^{\mathrm{s}}(\boldsymbol{x})=T_{\mathrm{A}}^{\mathrm{c}}(\boldsymbol{x})+r(\boldsymbol{x}),
$$

where $\boldsymbol{x}=\left[x_{1}, x_{2}, \ldots, x_{N}\right]^{T}$ indicates the dependence on the vector of parameters describing the physical reflectors. The correction term $r(\boldsymbol{x})$ is estimated by fitting a linear regression model on the residuals of the coarse and fine models, sampled over a limited training set $\boldsymbol{x}_{\boldsymbol{i}}$, as

$$
r_{i}\left(\boldsymbol{x}_{\boldsymbol{i}}\right)=T_{\mathrm{A}}\left(\boldsymbol{x}_{\boldsymbol{i}}\right)-T_{\mathrm{A}}^{\mathrm{c}}\left(\boldsymbol{x}_{\boldsymbol{i}}\right),
$$

where $x_{i}$ is selected as a star distribution to cover the full parameter space [13]. The correction term regression model assumes independent input variables (coupled variables may also be included if the number of samples are increased), and is limited to a second order polynomial of the form

$$
r(\boldsymbol{x})=\alpha_{0}+\sum_{n=1}^{N}\left[\alpha_{n} x_{n}+\beta_{n} x_{n}^{2}\right],
$$

trained, in a least squares sense, on $\boldsymbol{x}_{\boldsymbol{i}}$ to find the parameter vectors $\boldsymbol{\alpha}$ and $\boldsymbol{\beta}$ [14]. Using this regression model, the correction term $r(\boldsymbol{x})$ is generalized over the full parameter space from information collected at only a few samples $\boldsymbol{x}_{\boldsymbol{i}}$. $T_{\mathrm{A}}^{\mathrm{s}}$ is thus expected to be more accurate than $T_{\mathrm{A}}^{\mathrm{c}}$ over the full extent of $\boldsymbol{x}$.

\section{EXAMPLES}

To illustrate the method, examples of three types of reflector systems are investigated. The parameter sets for each case is chosen as representative of typical design and optimization scenarios, but in many real world scenarios different sets of parameters and constraints might be used. This is due to the fact that the mechanical design of the reflector surfaces often drives and constrains the electromagnetic design to a large degree. The brightness temperature model for $T_{\mathrm{b}}$ used in all cases is model 3 in [7]. This is the polarization averaged reduced expression of the full brightness temperature model, including atmospheric absorption by water and oxygen, galactic and cosmic microwave background emission, as well as ground emission and scattering, presented in [11, Sec. 4.3]. PO, augmented by Physical Theory of Diffraction (PTD), as implemented in the commercial code GRASP [15], is used as the solution method for the reflector systems. Near field effects are accounted for by using the spherical wave expansion of the feed radiation patterns. Simulations are performed on a laptop computer with a dual core $2.1 \mathrm{GHz}$ Intel $囚 \mathrm{Core}^{\mathrm{TM}}$ i74600U CPU and 8 GB memory. Recall that calculation of the main beam (and first few sidelobes) of a reflector system is significantly faster (typically at least an order of magnitude) than calculation of the full pattern over the $4 \pi$ sphere, as is required for antenna noise calculations done here. This is due to the smaller number of required field samples, but also due to the coarser mesh required to resolve the slow spatial variation of the current over the reflector surface for near broadside radiated fields. Other radiation pattern responses of interest, such as gain, sidelobe levels, and cross-polarization isolation, may thus be directly calculated using the full reflector system.

All temperature plots are shown as the average over $\boldsymbol{\theta}_{\mathrm{p}}$, calculated as ( $f$ dependence suppressed)

$$
\bar{T}_{\mathrm{A}}^{(*)}(\boldsymbol{x})=\frac{1}{N_{\mathrm{p}}} \sum_{t=1}^{N_{\mathrm{p}}} T_{\mathrm{A}}^{(*)}\left(\theta_{\mathrm{p} t}, \boldsymbol{x}\right),
$$

with the star symbol indicating any of $T_{\mathrm{A}}, T_{\mathrm{A}}^{(\mathrm{s})}$, or $T_{\mathrm{A}}^{(\mathrm{c})}$, and $\boldsymbol{\theta}_{\mathrm{p}}=\left[0^{\circ}, 3^{\circ}, 6^{\circ}, \ldots, 75^{\circ}\right]$ (giving $N_{\mathrm{p}}=26$ ). The accuracies of the coarse and surrogate models (indicated by superscripts ${ }^{\mathrm{c}}$ and ${ }^{\mathrm{s}}$ respectively) are evaluated on a validation set $\boldsymbol{x}_{\mathrm{v}}$ and described by error functions of the form

$$
\epsilon^{(\mathrm{c}, \mathrm{s})}\left(\boldsymbol{x}_{\mathrm{v}}\right)=\frac{\Delta^{(\mathrm{c}, \mathrm{s})}\left(\boldsymbol{x}_{\mathrm{v}}\right)}{\bar{T}_{\mathrm{A}}\left(\boldsymbol{x}_{\mathrm{v}}\right)}
$$

with

$$
\Delta^{(\mathrm{c}, \mathrm{s})}\left(\boldsymbol{x}_{\mathrm{v}}\right)=\left|\bar{T}_{\mathrm{A}}^{(\mathrm{c}, \mathrm{s})}\left(\boldsymbol{x}_{\mathrm{v}}\right)-\bar{T}_{\mathrm{A}}\left(\boldsymbol{x}_{\mathrm{v}}\right)\right| .
$$

Standard RMS errors, calculated over the full validation sets, are indicated as $\epsilon_{\mathrm{RMS}}^{(\mathrm{c}, \mathrm{s})}$. Furthermore, the maximum absolute differences between the fine model and the coarse and surrogate models respectively are also given and calculated as $\Delta_{\max }^{(\mathrm{c}, \mathrm{s})}=\max \left\{\Delta^{(\mathrm{c}, \mathrm{s})}\left(\boldsymbol{x}_{\mathrm{v}}\right)\right\}$.

\section{A. Prime Focus Paraboloid}

As a first example a prime focus fed paraboloid reflector system is investigated. The feed antenna is a low-gain axially corrugated horn, designed according to the equations provided in [16] for three corrugations, and simulated using the Methodof-Moments (MoM) in FEKO [17]. The reflector diameter is $D=40 \lambda$, with $\lambda$ indicating the wavelength. The focal length is varied in the range $F / D \in[0.35,0.5]$, with the training and validation sets chosen as $\boldsymbol{x}_{\boldsymbol{i}}=[0.35,0.43,0.5]$, $\boldsymbol{x}_{\boldsymbol{v}}=\left[\begin{array}{lll}0.35 & : 0.01: 0.5\end{array}\right]$. Feed and strut supports are neglected in the PO/PTD solution. The main effect of the feed and support blockage on $T_{\mathrm{A}}$ will be a ripple in the response as a function of $F / D$ because of the electrical length variation between the dish and the feed [18] - a so-called chromatic aberration. Since the exact structure of this ripple is frequency dependent, it is best neglected when doing an initial optimization or parametric study of $F$ to keep the goal function smooth and thus to avoid local optima.

Results for the PO/PTD simulations are shown in Fig. 2, where the reference MoM results, of the full structure consisting of the dish and feed, are also included. PO/PTD results are used for the fine model, and the surrogate model is seen to approximate the MoM result without the chromatic aberration ripple. The RMS error level for the coarse model (simulation time around 5 seconds per sample) is $\epsilon_{\mathrm{RMS}}^{(\mathrm{c})}=3.0 \%$ which is reduced to $\epsilon_{\mathrm{RMS}}^{(\mathrm{s})}=0.3 \%$ for the surrogate model. The maximum absolute differences are $\Delta_{\max }^{(\mathrm{c})}=0.91 \mathrm{~K}$ and $\Delta_{\max }^{(\mathrm{s})}=0.096 \mathrm{~K}$. Acceptable accuracy may be achieved using the coarse model directly, but the improvement achieved when using the surrogate is obvious. Only three fine model simulations (of around 20 seconds simulation time each) are required to construct the surrogate. 


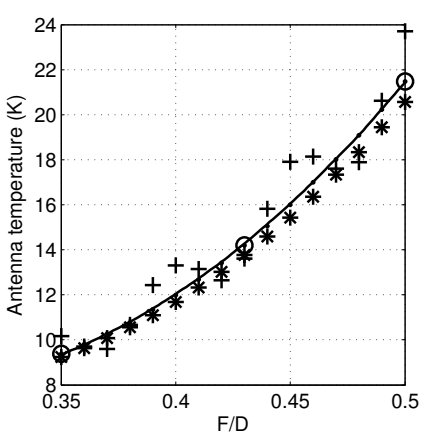

(a)

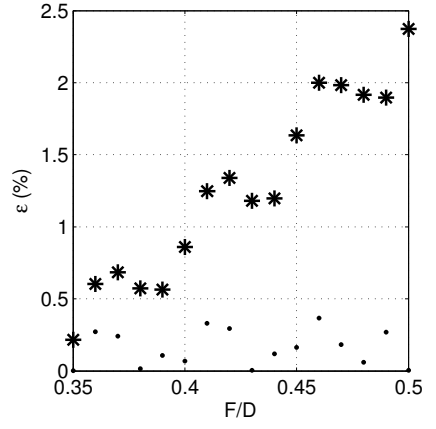

(b)
Fig. 2. Prime focus paraboloid antenna temperature results in (a), and modeling errors in (b). In (a) the solid line indicates the surrogate model temperature $\bar{T}_{\mathrm{A}}^{\mathrm{s}}(\boldsymbol{x})$, with the coarse and fine model verification sets $\bar{T}_{\mathrm{A}}^{\mathrm{c}}\left(\boldsymbol{x}_{\mathrm{v}}\right)$ and $\bar{T}_{\mathrm{A}}\left(\boldsymbol{x}_{\mathrm{V}}\right)$ indicated by black stars and red dots respectively (note that the dots lie almost exactly on the solid line). Red circles indicate the fine model training set $\bar{T}_{\mathrm{A}}\left(\boldsymbol{x}_{\mathrm{i}}\right)$, and crosses the MoM solution of $\bar{T}_{\mathrm{A}}\left(\boldsymbol{x}_{\mathrm{v}}\right)$. In (b) the coarse and surrogate model errors $\epsilon^{\mathrm{c}}\left(\boldsymbol{x}_{\mathrm{v}}\right)$ and $\epsilon^{\mathrm{s}}\left(\boldsymbol{x}_{\mathrm{v}}\right)$ are indicated by star and dot markers respectively.

\section{B. Symmetrical Cassegrain}

The next example investigates the response of a symmetrical Cassegrain system, described by the equations in [19], with the main reflector subtended angle fixed to $90^{\circ}$. Here an analytically defined axially symmetric Gaussian radiation pattern is assumed for the feed, with an edge taper value of $12 \mathrm{~dB}$ specified at $22^{\circ}$. The main reflector diameter is fixed at $D=100 \lambda$, since these types of reflector systems are normally significantly larger than prime focus types to reduce diffraction losses from the sub-reflector [20]. A twodimensional (2-D) parameter space is investigated over the range $x_{1}=d / D \in[0.05,0.14], x_{2}=\theta_{e} \in\left[17^{\circ}, 29^{\circ}\right]$. The training set is a star distribution (total of 5 samples), and the validation set a regular grid with 37 samples in $x_{1}$ and 13 samples in $x_{2}$.

Due to the large size of the reflectors the coarse model (simulation time around 5 seconds per sample; fine model around 5 minutes) RMS error is already at $\epsilon_{\mathrm{RMS}}^{(\mathrm{c})}=0.55 \%$, leaving little room for improvement in the surrogate model with a slightly worse $\epsilon_{\mathrm{RMS}}^{(\mathrm{s})}=0.70 \%$. The maximum absolute differences are $\Delta_{\max }^{(\mathrm{c})}=0.127 \mathrm{~K}$ and $\Delta_{\max }^{(\mathrm{s})}=0.126 \mathrm{~K}$. Note the ripple structure in the error response caused by the varying effective seperation distance between the two reflectors - an effect ignored in the coarse model.

\section{Shaped Offset Gregorian}

The final example considers a shaped offset Gregorian system, illuminated by the same horn feed as in Section III-A. The dishes are shaped, using the method described in [21] and [22, Sec. II], to produce a desired aperture distribution given a specified feed radiation pattern. Specifically, a Gaussian feed with $12 \mathrm{~dB}$ taper at $\theta_{e}$ is assumed, and the dishes are shaped to provide an aperture power density distribution of the form described in [23, II] i.e. a hybrid uniform/Gaussian distribution. This distribution is axially symmetric, with a central region of uniform power density. Outside this central region the power density decreases smoothly, as a Gaussian

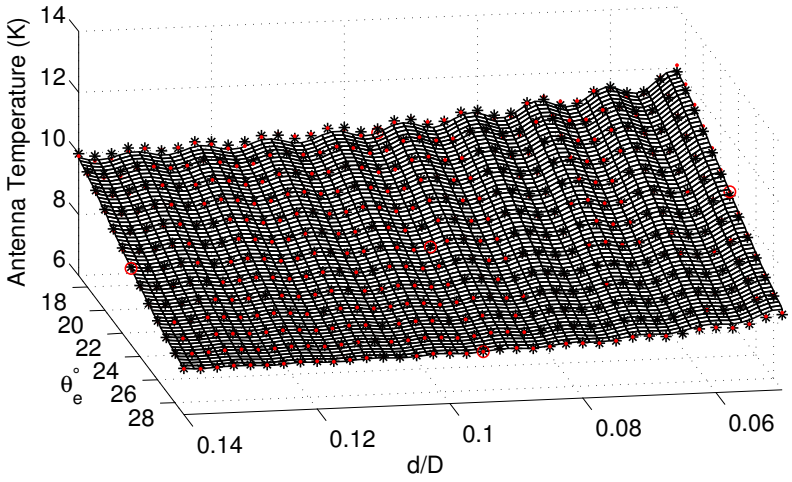

(a)

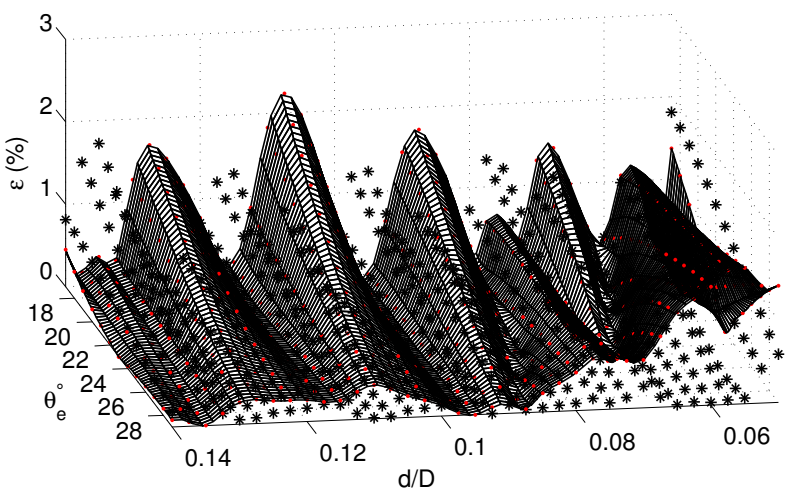

(b)

Fig. 3. Symmetrical Cassegrain antenna temperature results in (a), and modeling errors in (b). In (a) the spline interpolation surface indicates the surrogate model temperature $\bar{T}_{\mathrm{A}}^{\mathrm{s}}(\boldsymbol{x})$, with the coarse and fine model verification sets $\bar{T}_{\mathrm{A}}^{\mathrm{c}}\left(\boldsymbol{x}_{\mathrm{v}}\right)$ and $\bar{T}_{\mathrm{A}}\left(\boldsymbol{x}_{\mathrm{v}}\right)$ indicated by black stars and red dots respectively. Red circles indicate the fine model training set $\bar{T}_{\mathrm{A}}\left(\boldsymbol{x}_{\mathrm{i}}\right)$. In (b) the coarse and surrogate model errors $\epsilon^{\mathrm{c}}\left(\boldsymbol{x}_{\mathrm{V}}\right)$ and $\epsilon^{\mathrm{s}}\left(\boldsymbol{x}_{\mathrm{V}}\right)$ are indicated by star and dot markers respectively. A spline interpolation is used to generate the surface plot of $\epsilon^{\mathrm{s}}(\boldsymbol{x})$, which thus interpolates the red dots.

function, to a specified edge taper value. The parameter space of interest is again 2-D, with the first parameter describing the relative extent of the uniform aperture distribution $x_{1}=\rho_{\mathrm{MM}} \in[0,0.9]$, and the second the edge taper of the aperture distribution $x_{2}=b=[0 \mathrm{~dB}, 20 \mathrm{~dB}]$. These definitions are slightly different from those in [23]. The main reflector projected diameter is $D=50 \lambda$, and the maximum main and sub-reflector chord lengths are $60.67 \lambda$ and $16.67 \lambda$ respectively. The projected separation between the reflectors (onto a plane perpendicular to the main beam direction) is $1.67 \lambda$, and $\theta_{e}=58^{\circ}$. Results are shown in Fig. 4, with the training set again a star distribution of 5 samples, and the validation set a regular grid with $10^{2}$ samples.

This example shows a marked improvement in accuracy of the surrogate model when compared to the coarse model (simulation time around 5 seconds per sample; fine model around 90 seconds) from $\epsilon_{\mathrm{RMS}}^{(\mathrm{c})}=4.7 \%$ to $\epsilon_{\mathrm{RMS}}^{(\mathrm{s})}=1.0 \%$. The maximum absolute differences are $\Delta_{\max }^{(\mathrm{c})}=1.46 \mathrm{~K}$ and $\Delta_{\max }^{(\mathrm{s})}=0.36 \mathrm{~K}$. It is also worth noting that the coarse model is significantly more accurate in regions of low main reflector edge illumination $\left(b \rightarrow 20 \mathrm{~dB}\right.$ and $\left.\rho_{\mathrm{MM}} \rightarrow 0\right)$ due to the reduced edge diffraction. In the design of, for instance, radio telescopes with a large number of similar reflectors, such as the 


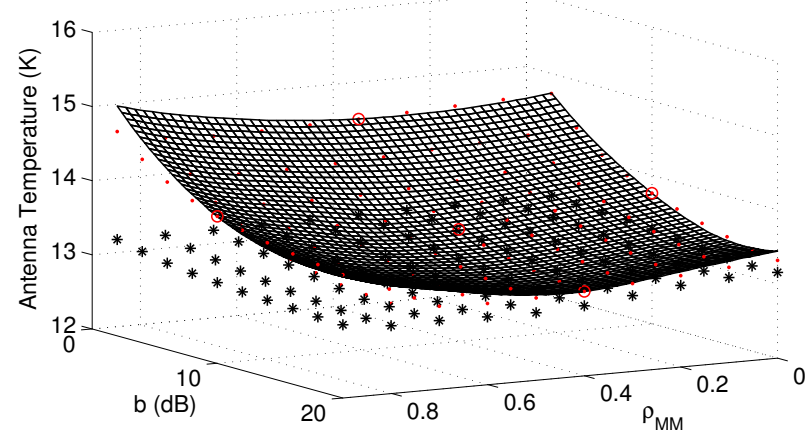

(a)

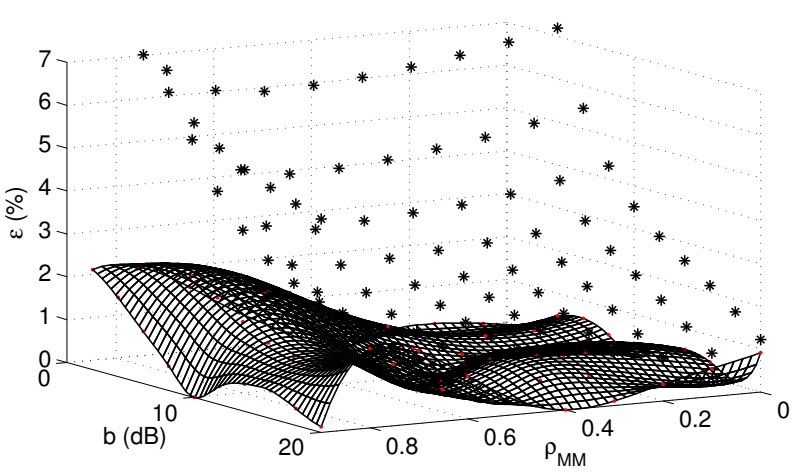

(b)

Fig. 4. Shaped offset Gregorian antenna temperature results in (a), and modeling errors in (b). In (a) the spline interpolation surface indicates the surrogate model temperature $\bar{T}_{\mathrm{A}}^{\mathrm{s}}(\boldsymbol{x})$, with the coarse and fine model verification sets $\bar{T}_{\mathrm{A}}^{\mathrm{c}}\left(\boldsymbol{x}_{\mathrm{v}}\right)$ and $\bar{T}_{\mathrm{A}}\left(\boldsymbol{x}_{\mathrm{V}}\right)$ indicated by black stars and red dots respectively. Red circles indicate the fine model training set $\bar{T}_{\mathrm{A}}\left(\boldsymbol{x}_{\mathrm{i}}\right)$. In (b) the coarse and surrogate model errors $\epsilon^{\mathrm{c}}\left(\boldsymbol{x}_{\mathrm{v}}\right)$ and $\epsilon^{\mathrm{S}}\left(\boldsymbol{x}_{\mathrm{V}}\right)$ are indicated by star and dot markers respectively. A spline interpolation is used to generate the surface plot of $\epsilon^{\mathrm{s}}(\boldsymbol{x})$, which thus interpolates the red dots.

SKA [3], such high levels of accuracy are critical in the design and optimization stage. In these systems - where hundreds or even thousands of dishes are to be manufactured and installed - a $1 \%$ improvement in receiving sensitivity of each the antenna systems translates to the equivalent of dozens (or even hundreds) fewer reflectors required to achieve the same level of receiving sensitivity.

\section{Summary AND CONCLUding REMARKS}

This communication presented a fast approximation, valid over large design parameters spaces, to the antenna noise temperature contribution from background radiometric noise in reflector antenna systems. The main reflector is removed from the calculation domain to speed up the analysis, and accuracy is retained by alignment of this coarse model response to fine model responses calculated over a sparse set of training points in the parameter space. Various types of reflector configurations were evaluated using the suggested method, and accuracy of better than $1 \%$ is obtained at speed-ups of more than an order of magnitude. Noise contributions due to the reflector surfaces (conduction losses and scattering due to surface errors), which become more apparent at higher frequencies, are not considered here. This will be the subject of future research, where it is foreseen that a similar method may be used for the fast and accurate parametric modeling of the noise temperature contribution from the reflector surfaces.

\section{REFERENCES}

[1] T. Y. Otoshi, Noise Temperature Theory and Applications for Deep Space Communications Antenna Systems. Artech House, 2008.

[2] J. L. Jonas, "MeerKAT - The South African array with composite dishes and wide-band single pixel feeds," Proc. IEEE, vol. 97, no. 8, pp. 1522 1530, Aug. 2009.

[3] P. E. Dewdney, P. J. Hall, R. T. Schilizzi, and T. J. L. W. Lazio, "The Square Kilometer Array," Proc. IEEE, vol. 97, no. 8, pp. 1482 - 1496, Aug. 2009.

[4] P.-S. Kildal, S. A. Skyttemyr, and A. A. Kishk, "G/T maximization of a paraboloidal reflector fed by a dipole-disk antenna with ring by using the multiple-reflection approach and the moment method," IEEE Trans. Antennas Propag., vol. 45, no. 7, pp. 1130-1139, Jul. 1997.

[5] W. A. Imbriale, "Faster antenna noise temperature calculations using a novel approximation technique," in Proc. IEEE Int. Symp. Antennas and Propag., Toronto, ON, Canada, Jul. 2010, pp. 1 - 4.

[6] D. I. L. de Villiers and R. Lehmensiek, "Efficient simulation of radiometric noise in offset Gregorian antenna systems," in Proc. 7th Eur. Conf. Antennas and Propag. (EuCAP), Gothenburg, Sweden, Apr. 2013, pp. $3357-3359$.

[7] — "Rapid calculation of antenna noise temperature in offset Gregorian reflector systems," IEEE Trans. Antennas Propag., vol. 63, no. 4, pp. 1564-1571, Apr. 2015.

[8] R. Lehmensiek and D. I. L. De Villiers, "On noise temperature calculations of offset Gregorian reflector systems," in Proc. Int. Conf. Electromagn. Adv. Appl. (ICEAA), Turin, Italy, Sep. 2015.

[9] S. Srikanth, "Comparison of spillover loss of offset Gregorian and Cassegrain antennas," in Proc. IEEE Int. Symp. Antennas and Propag., vol. 1, Jun. 1991, pp. 444-447.

[10] S. Koziel and L. Leifsson, Eds., Surrogate-Based Modeling and Optimization. Applications in Engineering. Springer, 2013.

[11] G. C. Medellin, "Antenna noise temperature calculations," in SKA Memo 95, Jul. 2007. [Online]. Available: https://cornell.academia.edu/ GermanCortes

[12] O. Borries, H. H. Viskum, P. Meincke, E. Jørgensen, P. C. Hansen, and C. H. Schmidt, "Analysis of electrically large antennas using fast physical optics," in Proc. Eur. Conf. Antennas and Propag. (EuCAP), Lisbon, Portugal, Apr. 2015.

[13] J. W. Bandler, Q. S. Cheng, S. A. Dakroury, A. S. Mohamed, M. H. Bakr, K. Madsen, and J. Søndergaard, "Space mapping: the state of the art," IEEE Trans. Microw. Theory Tech., vol. 52, no. 1, pp. 337-361, Jan. 2004.

[14] A. F. Seber and A. J. Lee, Linear Regression Analysis, 2nd ed. Wiley, 2003.

[15] TICRA, Copenhagen, Denmark. GRASP10, Version 10.4.0. [Online]. Available: www.ticra.com

[16] C. Granet, G. L. James, and A. R. Forsyth, Modern Antenna Handbook. John Wiley \& Sons, 2008, ch. 3.4.6, pp. 127-129.

[17] Altair Development S.A. (Pty) Ltd, Stellenbosch, South Africa. FEKO, Suite 7.0. [Online]. Available: www.feko.info

[18] D. Morris, "Chromatism in radio telescopes due to blocking and feed scattering," Astron. Astrophysics, vol. 67, pp. 221-228, Jul. 1978.

[19] C. Granet, "Designing axially symmetric Cassegrain or Gregorian dualreflector antennas from combinations of prescribed geometric parameters," IEEE Antennas Propag. Mag., vol. 40, no. 2, pp. 76-82, Apr. 1998.

[20] P.-S. Kildal, "The effects of subreflector diffraction on the aperture efficiency of a conventional Cassegrain antenna - an analytical approach," IEEE Trans. Antennas Prapag., vol. AP-31, no. 6, pp. 903-909, Nov. 1983.

[21] — "Synthesis of multireflector antennas by kinematic and dynamic ray tracing," IEEE Trans. Antennas Propag., vol. 38, no. 10, pp. 15871599, Oct. 1990.

[22] R. Lehmensiek, I. P. Theron, and D. I. L. de Villiers, "Deriving an optimum mapping function for the SKA shaped offset Gregorian reflectors," IEEE Trans. Antennas Propag., vol. 63, no. 11, pp. 46584666, Nov. 2015

[23] V. Galindo-Israel, W. Veruttipong, R. D. Norrod, and W. A. Imbriale, "Scanning properties of large dual-shaped offset and symmetric reflector antennas,' IEEE Trans. Antennas Propag., vol. 40, no. 4, pp. 422-432, Apr. 1992. 